\title{
Irr regulates brucebactin and 2,3-dihydroxybenzoic acid biosynthesis, and is implicated in the oxidative stress resistance and intracellular survival of Brucella abortus
}

Correspondence

Marta Almirón

malmiron@iib.unsam.edu.ar

Received 19 December 2005

Revised 31 May 2006

Accepted 31 May 2006

\author{
Marcela Martínez, Rodolfo A. Ugalde and Marta Almirón
}

Instituto de Investigaciones Biotecnológicas, Instituto Tecnológico de Chascomús (IIB, INTECH), Consejo Nacional de Investigaciones Científicas y Técnicas, Universidad Nacional de General San Martín (CONICET-UNSAM), San Martín 1650, Argentina

\begin{abstract}
Brucella abortus faces iron deprivation in both nature and the host. To overcome this limitation, Brucella secretes the siderophores 2,3-dihydroxybenzoic acid and brucebactin. A Fur-like protein named Irr has previously been characterized in B. abortus; this protein is present in the $\alpha-2$ group of Proteobacteria only, where it negatively regulates haem biosynthesis when iron is scarce. Additional evidence that Irr also regulates the synthesis of both siderophores is presented here. Transcriptional lacZ fusion and chemical determinations revealed that Irr induced the transcription of the operon involved in the synthesis of the catecholic siderophores, which were consequently secreted under conditions of iron limitation. Irr was able to bind the upstream region of the operon, as shown by electrophoretic mobility shift assay. A B. abortus irr mutant showed higher intracellular haem content, catalase activity and resistance to hydrogen peroxide than the wild-type strain. The mutation also improved the replication and survival of iron-depleted bacteria within cultured mammalian cells. Although the pathogenesis of Brucella correlates with its ability to replicate intracellularly, pathogenicity was not attenuated when assayed in a murine model.
\end{abstract}

\section{INTRODUCTION}

Iron is an essential micronutrient for almost all living organisms. In nature, iron is mainly present in a ferric insoluble state, with reduced biological availability. One of the strategies developed by bacteria to acquire iron under restrictive conditions is the synthesis of low-molecular-mass iron chelators, known as siderophores, together with the receptors for the internalization of ferrisiderophores (Wandersman \& Delepelaire, 2004). This iron acquisition mechanism is frequently linked to the virulence of bacteria because it contributes to the establishment of a successful infection (Litwin \& Calderwood, 1993). In contrast, a high concentration of iron is toxic, due to its participation in reactions that generate reactive oxygen species, which can lead to cellular damage. In these conditions, bacteria usually induce the synthesis of storage proteins and oxidative stress enzymes that remove toxic molecules. According to this scenario, the tight regulation of iron homeostasis is fundamental for bacterial life.

Brucella abortus is the aetiological agent of bovine brucellosis, which is an infection of humans and cattle.

Abbreviations: CAS, chrome azurol S; 2,3-DHBA, 2,3-dihydroxybenzoic acid; DIP, 2,2'-dipyridyl; EMSA, electrophoretic mobility shift assay; p.i., post-infection.
The organism lives mainly intracellularly, and its pathogenesis correlates with the ability to invade and replicate within professional and non-professional phagocytes (Smith \& Ficht, 1990). During infection, iron availability is reduced as part of the host defence against micro-organisms, implying that B. abortus faces iron limitation in this process (Bullen \& Griffiths, 1999). Under conditions of iron depletion, B. abortus secretes the two catecholic siderophores brucebactin and 2,3-dihydroxybenzoic acid (2,3-DHBA), which have been associated with virulence of the species in ruminants, suggesting the importance of this mechanism for iron acquisition in the natural host (Bellaire et al., 2003). Brucebactin, whose structure remains unknown, has recently been reported to be the most active siderophore of B. abortus (Gonzalez Carrero et al., 2002); it is produced from 2,3DHBA through a poorly understood pathway. Biosynthesis of 2,3-DHBA requires genes organized in the $d h b C E B A$ operon, whose expression is regulated by iron (Bellaire et al., 2003). Even though two Fur boxes have been identified in the promoter region, the ferric-uptake regulator Fur does not repress transcription of this operon (Roop et al., 2004). No additional data on this regulation have been reported heretofore.

We have recently characterized the iron response regulator Irr from B. abortus, and it belongs to the Fur family 
(Martínez et al., 2005). Irr downregulates haem biosynthesis when iron is insufficient. This biosynthesis involves six sequential enzymic reactions leading to protoporphyrin IX. In the last step of the pathway, ferrochelatase inserts ferrous iron into the porphyrin ring to yield haem. The interruption of this step is detrimental for B. abortus virulence (Almirón et al., 2001). Hence, considering the role of Irr in the regulation of an iron-demanding pathway implicated in $B$. abortus virulence, we decided to investigate whether Irr controls iron-uptake systems, and whether it plays any role during infection. Here, we present evidence that Irr directly induces the biosynthesis of 2,3-DHBA and brucebactin, but that it is not required for virulence in mice. Interestingly, under conditions of iron limitation, a mutant lacking Irr displayed increased resistance to hydrogen peroxide due to higher levels of haem and catalase activity than the wild-type strain. Compared with the wild-type, the mutant showed improved intracellular replication and survival inside HeLa and J774 cells, thus implicating Irr in the pathogenesis of $B$. abortus.

\section{METHODS}

Bacterial strains and growth conditions. B. abortus strains 2308, 2308IK (2308irr:: km), 2308IK(pBBRirr) (the mutant complemented with the wild-type irr gene cloned into pBBR1MCS-4) and 2308C (2308dhbC:: lacZ) were obtained from the laboratory stock (Martínez et al., 2005). A double mutant 2308IC (2308irr:: km, dhbC::lacZ), which carries the chromosomal mutations of $2308 \mathrm{IK}$ and 2308C, was constructed for this study by gene replacement (Martínez et al., 2005). All bacterial liquid cultures were incubated at $37^{\circ} \mathrm{C}$ in a rotary shaker at 250 r.p.m. Luria-Bertani medium containing the iron chelator 2,2'-dipyridyl (LB-DIP; $0.45 \mathrm{mM}$ ), and modified Gerhardt's (MG) medium, were used as iron-deficient media (Lopez-Goñi et al., 1992). When required, the media were supplemented with $100 \mu \mathrm{g}$ ampicillin $\mathrm{ml}^{-1}$ or $50 \mu \mathrm{g}$ kanamycin $\mathrm{ml}^{-1}$. Procedures using live brucellae were performed in a biosafety level 3 laboratory. All the reagents were purchased from Sigma, unless otherwise stated.

$\boldsymbol{\beta}$-Galactosidase enzyme assay. Levels of $\beta$-galactosidase were measured from liquid iron-depleted cultures as described by Miller (1992).

\section{Siderophore detection}

The chrome azurol S (CAS) assay. This assay was used to determine brucebactin secretion only, since different amounts of commercial 2,3-DHBA had no effect on the absorbance of the CAS reagent, either in plate or in liquid cultures. CAS agar plates were prepared according to the method of Schwyn \& Neilands (1987), with the following modifications: (i) MG was used instead of MM9 medium, (ii) the $\mathrm{K}_{2} \mathrm{HPO}_{4}$ concentration was reduced to $0.3 \mathrm{~g} \mathrm{l}^{-1}$, and (iii) glucose was added in place of glycerol and lactic acid to avoid interference of these compounds with CAS (Gonzalez Carrero et al., 2002). Cells grown for $24 \mathrm{~h}$ in $1 \mathrm{ml} \mathrm{MG}$ were washed and resuspended in $10 \mu \mathrm{l}$ PBS, which was applied to a sterile filter disk on the CAS plate. The halo produced around the spot was observed after $72 \mathrm{~h}$ incubation at $37^{\circ} \mathrm{C}$. For detection in liquid cultures, the supernatants were mixed with an equal volume of CAS reagent, and the absorbance was read at $630 \mathrm{~nm}$. Percentage siderophore activity was calculated by using the formula $[(X-Y) / X] \times 100$, where $X$ and $Y$ are the absorbance values of the growth medium and the sample supernatant, respectively. In order to set up the experimental conditions for catechol detection, measurements were taken at different time points during the incubation of cells in MG medium. Maximal differences were obtained when the cultures reached $\mathrm{OD}_{600}$ $\sim 1$. No catechol was detected when determinations were made from supernatants of MG cultures supplemented with $200 \mu \mathrm{M}$ ferric citrate.

HPLC analysis. This was performed to detect 2,3-DHBA. Supernatants $(10 \mathrm{ml})$ from the bacterial cultures assayed for brucebactin were acidified to $\mathrm{pH} 2 \cdot 0$ with $6 \mathrm{M} \mathrm{HCl}$. Acidified samples were extracted with ethyl acetate $\left[20 \mathrm{ml}(100 \mathrm{ml} \text { supernatant })^{-1}\right]$. The catechol-containing extracts were concentrated in a vacuum system, and $0.2 \mathrm{ml}$ of each extract was fractionated on a C18 Sephasil peptide reverse-phase column $(12 \mu \mathrm{M}, 4.6 \times 250 \mathrm{~mm}$; Pharmacia Biotech). The procedure was performed using a gradient of $10-50 \%$ acetonitrile in water, with $0 \cdot 1 \%$ trifluoroacetic acid at a constant flow rate of $1 \mathrm{ml} \mathrm{min}^{-1}$. The column profile was monitored by following the absorbance at $254 \mathrm{~nm}$. Commercial 2,3-DHBA dissolved in ethyl acetate was employed as a standard.

Haem determination. The intracellular concentration of haem in B. abortus strains was determined as described by Frustaci et al. (1991). Commercial haemin was used as a standard in the range $10-200 \mathrm{nM}$, and it was assayed at the same time as the B. abortus samples.

Catalase assay. B. abortus strains were grown to $\mathrm{OD}_{600} \sim 1 \cdot 0$ in LB-DIP. Cells were centrifuged, washed, and resuspended in PBS. Suspensions were sonicated, and further centrifuged $(20000 \mathrm{~g}$ for $10 \mathrm{~min}$ at $\left.4{ }^{\circ} \mathrm{C}\right)$. Aliquots from the supernatants were assayed for catalase activity, which was determined by following the decomposition of $18 \mathrm{mM}$ hydrogen peroxide at $240 \mathrm{~nm}$ (Beers \& Sizer, 1952). One unit of activity was defined as the amount of enzyme that catalysed the decomposition of one micromole of hydrogen peroxide per minute. Protein concentration was determined as described by Bradford (1976).

Hydrogen peroxide sensitivity assay. Cells were grown in either LB or LB-DIP to the exponential or stationary phase of growth. Cells were centrifuged (20000 $\mathrm{g}$ for $10 \mathrm{~min}$ ), washed, and diluted 1 in 10 in PBS. Aliquots from the logarithmic- and stationary-phase suspensions were challenged with 33 and $100 \mathrm{mM}$ hydrogen peroxide, respectively. Assays were carried out at room temperature, without shaking. At different time points, samples were taken, and these were serially diluted, and plated on LB plates. Colonies were counted after $48 \mathrm{~h}$ at $37^{\circ} \mathrm{C}$. Survival rates at different time points were determined as percentages of the number of colonies in the original inoculum.

DNA-binding assay. The ability of the recombinant Irr protein to bind to the upstream $d h b C E B A$ region was determined by an electrophoretic mobility shift assay (EMSA). A $0.62 \mathrm{~kb}$ DNA fragment was PCR-amplified with the sense primer (5'-GCTCTAGACCTGTCCCGGCGCAG- $\left.3^{\prime}\right)$ and the antisense primer $\left(5^{\prime}\right.$-TTCTGCAGTTGCCGCTGGCCGCCA-3'). The PCR product was digested with HindIII, and the $0.24 \mathrm{~kb}$ fragment containing the two promoter regions was purified from an agarose gel. Samples containing $0 \cdot 7 \mu \mathrm{g}$ of this DNA were mixed with $0,0.6$ or $0.6 \mathrm{nmol}$ of the recombinant Irr in $1 \times$ binding buffer $(20 \mathrm{mM}$ Tris, $\mathrm{pH} 7 \cdot 8,5 \%$ glycerol, v/v, $1 \mathrm{mM} \mathrm{DTT}, 50 \mu \mathrm{g} \mathrm{BSA} \mathrm{ml}^{-1}$, and $5 \mu \mathrm{g}$ salmon sperm DNA ml${ }^{-1}$ ). The samples were then incubated at room temperature for $15 \mathrm{~min}$. When needed, $3 \mu \mathrm{l}$ polyclonal mouse anti-Irr serum was added to the reaction mix, which was incubated for another $15 \mathrm{~min}$. As a control, a $0.76 \mathrm{~kb}$ chromosomal DNA fragment was used; this was amplified by PCR with the sense primer (5'-CGGGATCCTCGTGTTCGCAGGCTCA-3') and the antisense primer (5'-TGCACTGCAGTTCGGGAGGACGAAT-3'). EMSA reactions were analysed on 
$1.5 \%$ agarose gel in $0.5 \times$ Tris/borate/EDTA buffer. After the electrophoresis, the gel was stained with $0.5 \mu \mathrm{g}$ ethidium bromide $\mathrm{ml}^{-1}$, and visualized under UV light.

Intracellular Brucella survival experiment. Infection of HeLa and murine-macrophage-like J774 cell lines was performed as previously described (Almirón et al., 2001), with the following modifications: (i) bacterial strains were grown in either LB or LB-DIP; (ii) during infection, no fetal bovine serum was added to the cell medium in order to avoid any possible iron acquisition; and (iii) at $1 \mathrm{~h}$ post-infection (p.i.), when non-adherent bacteria had been eliminated, infected eukaryotic cells were incubated with the appropriate medium supplemented with fetal bovine serum.

In vivo experimental infection. Eight-week-old female $\mathrm{BALB} / \mathrm{C}$ mice were injected intraperitoneally with $0.1 \mathrm{ml}$ of a bacterial suspension prepared in PBS (about $10^{4}$ c.f.u. grown in LB-DIP). At 1 and 3 weeks p.i., an excess of ether anaesthesia was administered, and mice were bled to death by cardiac puncture. The spleen was aseptically dissected, weighed, and then homogenized in PBS. The number of viable bacteria was determined by plating serial dilutions on LB agar.

Statistical analysis. All statistical analysis was performed using Student's two-tailed $t$ test. $P$ values $\leqslant 0.05$ were considered significant. Results are expressed as means $\pm \mathrm{SD}$.

\section{RESULTS}

\section{Analysis of secreted siderophores}

Each siderophore released by Brucella was detected individually, as described in Methods. Brucebactin was assayed directly with CAS. As shown in Fig. 1(a), after 3 days incubation, the mutant $B$. abortus 2308IK secreted less brucebactin than the wild-type 2308, as judged by the size of the halo. Brucebactin production of the mutant was restored when complemented with the wild-type irr, thus confirming that Irr was responsible for the observed phenotype.

To detect secreted 2,3-DHBA, strains 2308, 2308IK and $2308 \mathrm{IK}$ (pBBRirr) were assayed together with B. abortus 2308C, a strain that does not produce siderophores. Supernatants of cultures were analysed by reverse-phase HPLC, and the area of the compound corresponding to the 2,3-DHBA standard was used to determine the amount of 2,3-DHBA secretion. As shown in Fig. 1(b), the B. abortus wild-type and the complemented strain secreted three- to fivefold more 2,3-DHBA than the mutant B. abortus 2308IK. To compare secretion of the catecholic compounds in the same medium, the relative activity of brucebactin was determined in liquid samples, as described in Methods. In agreement with the above result, the parental strain secreted over twofold more brucebactin than the irr mutant. The relative siderophore activities determined in the supernatants of 2308 and $2308 \mathrm{IK}$ from three independent experiments performed in duplicate were $87 \pm 1$ and $29 \pm 6 \%$, respectively $(P<0 \cdot 05)$. The mutant phenotype was also reverted by complementation with the wild-type irr gene to give $76 \pm 11 \%$ activity, against $1 \%$ obtained in the (a)

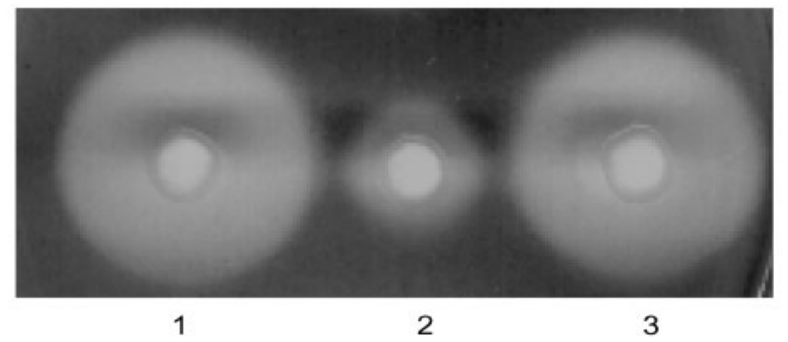

(b)

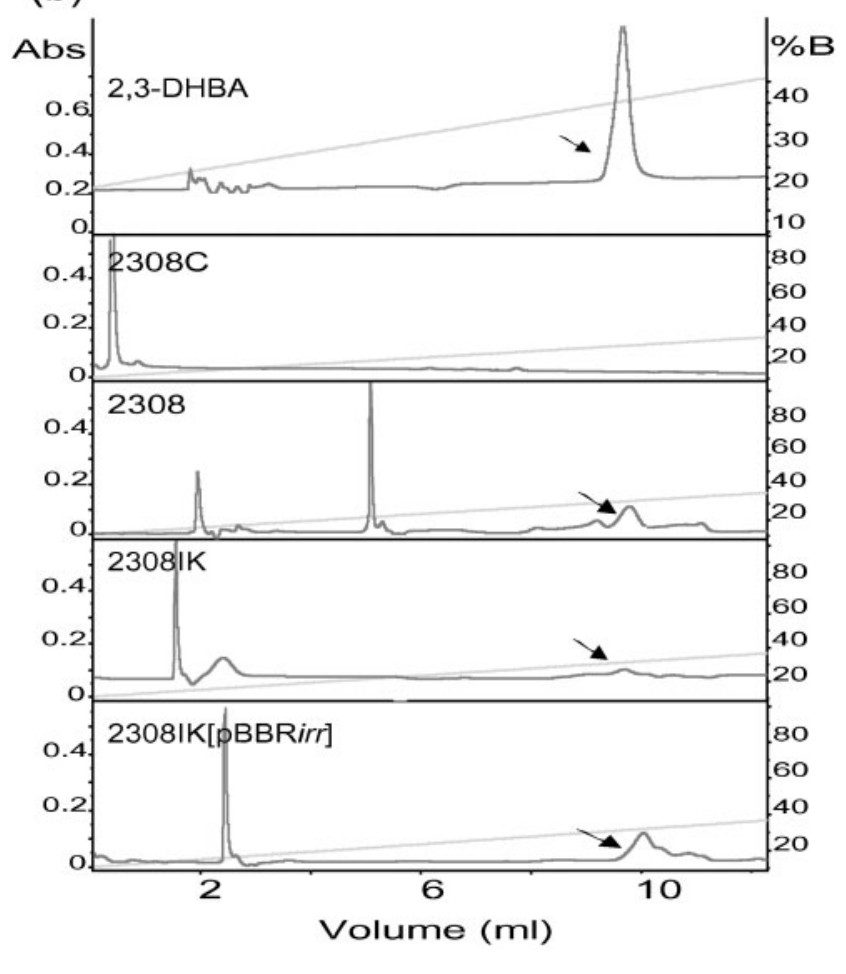

Fig. 1. Siderophore analysis from iron-restricted $B$. abortus cultures. (a) Detection of brucebactin secretion on CAS plates. $B$. abortus cultures were applied to filter disks on CAS plates, and incubated for $48-72 \mathrm{~h}$ at $37^{\circ} \mathrm{C}$. Brucebactin was detected as an orange halo around 2308 (1), 2308/K (2), and $2308 \mathrm{IK}(\mathrm{pBBR} i r$ ) (3). (b) Detection of 2,3-DHBA (arrow) in ethyl acetate extracts of supernatants assessed by reversephase HPLC. The column was eluted with a linear gradient of $10-50 \%$ acetonitrile (\%B), and absorbance (Abs) was recorded at a wavelength of $254 \mathrm{~nm}$.

supernatant of 2308C. Therefore, active Irr increased the amount of catecholic siderophores secreted by B. abortus.

\section{Effect of Irr on the expression of the dhbCEBA operon}

In order to determine whether the higher amounts of the secreted siderophores were the consequence of induced transcription of the genes involved in their synthesis, a chromosomal $d h b C-l a c Z$ fusion was analysed in the 
background of the parental and irr mutant strains, 2308C and 2308IC, respectively. The $\beta$-galactosidase activity of the $2308 \mathrm{C}$ strain ( $1252 \pm 140$ Miller units) was about twofold higher than that of the strain 2308IC carrying the irr mutation $(609 \pm 72$ Miller units) $(P<0 \cdot 05)$. These results were obtained from three independent experiments performed in duplicate. No $\beta$-galactosidase activity was obtained when the experiments were done either with cultures at $\mathrm{OD}_{600}<0 \cdot 4$, or with MG cultures supplemented with ferric citrate. Maximal induction of transcription was observed during the stationary growth phase of iron-limited cultures. These data corresponded to the decreased secretion of siderophores in the absence of Irr, suggesting that Irr contributes to positive transcriptional regulation of the operon when cells are growing under conditions of iron limitation.

To investigate the interaction of Irr with DNA, gel mobility shift assays were performed, as shown in Fig. 2. The fragment of DNA containing the two promoters of the dhbCEBA operon was mixed with the recombinant Irr protein, as indicated in Methods. The DNA mobility was retarded in the

(a)

aagcttcgttcagcgtcggecaatcacceggttgacaaattgggacca attaaatcaagtataaa acgtgactgattettcaagtattaatttc ggcaacgacgccaaggagactcccegtgagaacgagcgecetgaaa tacceaacttcagacgcagaccatcttattgaaaataätacttat $t$ ctcgtttca

(b)

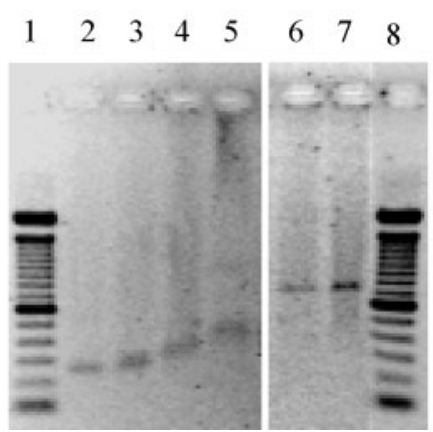

Fig. 2. DNA binding of Irr. (a) Nucleotide sequence of the dhbCEBA promoter region used in EMSA. The -35 and -10 regions of the two promoters are underlined, and the putative ICE-like motifs are indicated in bold. (b) Gel retardation assay. The DNA fragment shown in (a) was run in the absence (lane 2 ), and in the presence of 0.06 (lane 3) and $0.6 \mathrm{nmol}$ (lane 4) recombinant Irr protein. Anti-Irr serum was added to the complex shown in lane 4 (lane 5). An unrelated DNA fragment was run in the absence (lane 6) and presence of $0.6 \mathrm{nmol}$ Irr (lane 7). Ladders of $100 \mathrm{bp}$ were run in lanes 1 and 8 as molecular mass markers. After electrophoresis, the $1.5 \%$ agarose gel was stained with ethidium bromide. A negative image of the gel is shown for clarity. presence of increasing concentrations of Irr (Fig. 2b, lanes 3 and 4), and also when the anti-Irr serum was added to the reaction mix (Fig. 2b, lane 5). Even though the shift observed in the DNA mobility was slight, it was highly reproducible. This binding seems to be specific for two reasons. First, the non-specific binding of Irr was minimized by the addition of salmon-sperm DNA to the binding buffer. Second, the mobility of a DNA fragment without the ICE motif was not retarded in the presence of the maximal amount of Irr protein used in our experimental conditions (Fig. 2b, lanes 6 and 7). The extent of retardation could not be further improved by use of different binding buffers, native PAGE, or manipulation of the agarose concentration.

\section{Oxidative stress response}

Bearing in mind the relation between iron and oxidation, the phenotype of the irr mutant was investigated under oxidative stress. Preliminary results obtained from LB and LB-DIP plates with disks containing different concentrations of hydrogen peroxide showed a discernible phenotype between the wild-type and the mutant when cells were grown on LB-DIP. Thus, the sensitivity to this oxidative agent was investigated by measuring the percentage survival from cultures growing in liquid iron-restricted medium, using control cultures grown in LB. Since Gram-negative bacteria are more resistant to hydrogen peroxide during the stationary phase of growth than during the exponential phase (Almirón et al., 1992), logarithmic B. abortus 2308 and 2308IK cells were challenged with $33 \mathrm{mM}$ hydrogen peroxide, and stationary-phase cells with $100 \mathrm{mM}$ hydrogen peroxide. As shown in Fig. 3, mutant cells were more resistant than the wild-type when grown in iron-deficient medium. Between 40 and $60 \%$ of the mutant population survived after $35 \mathrm{~min}$ treatment when cells were in exponential phase (Fig. 3a), and after $15 \mathrm{~min}$ when they were in stationary phase (Fig. 3b). At similar time points, less than $10 \%$ of the wild-type population survived. After 60 min exposure, while both logarithmic and stationary $B$. abortus 2308 cells could not recover from the imposed stress condition, mutant cells remained viable. Non-viable cells were recovered after $15 \mathrm{~min}$ exposure to 33 or $100 \mathrm{mM}$ hydrogen peroxide when the experiment was done with logarithmic- or stationary-phase cells grown in LB medium (data from four independent experiments).

Catalase is the enzyme that inactivates hydrogen peroxide, and Brucella has one catalase encoded by katE (Sha et al., 1994). Thus, we determined the specific activity of catalase in the B. abortus strains. The B. abortus irr mutant expressed significantly more catalase activity than the wild-type. Values obtained from three independent experiments performed when the cultures reached $\mathrm{OD}_{600} \sim 1$ were $562 \pm 85$ and $47 \pm 5$ units $\mathrm{mg}^{-1}$ for $2308 \mathrm{IK}$ and 2308 , respectively $(P<0 \cdot 05)$. These data support the survival phenotype shown in Fig. 3.

As catalase is a haemoprotein, and we have previously shown that the B. abortus irr mutant accumulates haem precursors 

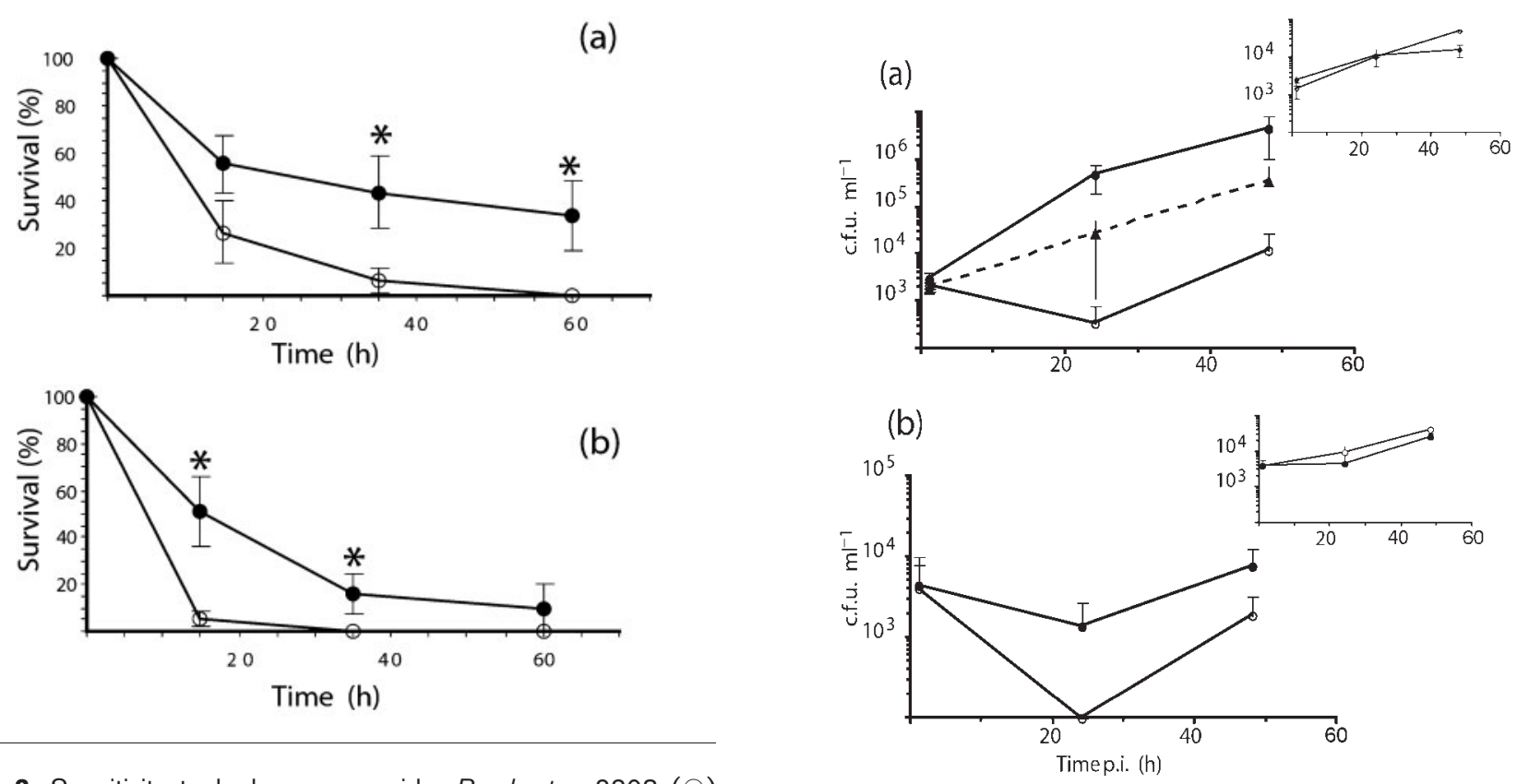

Fig. 3. Sensitivity to hydrogen peroxide. B. abortus $2308(\bigcirc)$ and $2308 \mathrm{IK}(0)$ cells were grown in LB-DIP, and treated with $33 \mathrm{mM}$ during the exponential phase of growth (a), and with $100 \mathrm{mM}$ hydrogen peroxide during stationary phase (b). The number of c.f.u. $\mathrm{ml}^{-1}$ recovered at different time points was related to the initial number of viable cells (about $1 \times 10^{8}$ c.f.u. $\mathrm{ml}^{-1}$ ), and expressed as a percentage. Data are means \pm SD of three independent experiments. ${ }^{*} P<0.05$.

under iron limitation (Martínez et al., 2005), we investigated whether there was a higher level of haem in mutant cells that could account for the higher catalase activity observed. Increases of more than twofold in the haemin intracellular concentration of $B$. abortus $2308 \mathrm{IK}$ were obtained in comparison with 2308 when the cells were grown in MG. The data obtained from three independent experiments were $15 \cdot 4 \pm 3 \cdot 6$ and $40 \cdot 2 \pm 8 \cdot 1 \mathrm{nM}(P<0 \cdot 05)$ for the wildtype and the mutant cells, respectively; data obtained from three independent experiments repeated in LB-DIP medium were in agreement with these results: $48 \cdot 2 \pm 1 \cdot 1$ and $80 \cdot 8 \pm 3 \cdot 1 \mathrm{nM}$ for wild-type and mutant cells, respectively; $P<0 \cdot 05$.

\section{Intracellular survival}

The capacity to invade and replicate inside HeLa and J774 cell lines was assayed with 2308 and 2308IK grown in irondeficient and iron-sufficient media, and 2308IK(pBBRirr) grown in iron-deficient medium. Cell invasion of HeLa cells (Fig. 4a) and the macrophage-like J774 cells (Fig. 4b) was similar among the strains tested. However, at $24 \mathrm{~h}$ p.i. of bacteria grown in iron-deficient medium, it was observed that while $2308 \mathrm{IK}$ was able to replicate inside HeLa cells, 2308 showed a decrease in the number of viable intracellular bacteria. At 48 h p.i., both strains were replicating. As shown in the inset, when bacteria were grown in iron-sufficient medium, this decline was not observed for the wild-type.

Fig. 4. Intracellular survival of $B$. abortus strains in HeLa (a) and $J 774$ (b) cell lines. (a) Cells were infected with $B$. abortus $2308(\bigcirc)$, 2308IK ( $)$ and 2308(pBBRirr) $(\boldsymbol{\Delta})$ at an m.o.i. of 100 after growth under iron limitation. At different times p.i., eukaryotic cells were lysed, and the number of viable intracellular bacteria was determined. The inset shows, using the same units, the infection with B. abortus 2308 and 2308IK grown without iron limitation. (b) $\mathbf{J} 774$ cells were infected at an m.o.i. of 50 with B. abortus $2308(\bigcirc)$ and $2308 \mathrm{KK}(\bullet)$ grown under conditions of iron limitation. The inset represents the infection with the same strains grown without iron limitation. Data are expressed as means $\pm S D$ of three independent experiments.

Thus, to investigate whether the enhanced replication of 2308IK at $24 \mathrm{~h}$ p.i. was due to the absence of Irr, 2308IK(pBBRirr) was assayed under the same conditions. This complemented strain behaved in the same way as the wild-type, suggesting that the phenotype was a consequence of the mutation in the irr gene. When J774 cells were infected with 2308 or $2308 \mathrm{IK}$, an initial reduction in the number of viable intracellular bacteria was detected at $10 \mathrm{~h}$ p.i. Whilst 2308IK showed replication at 24 and 48 h p.i., the viability of 2308 cells was seriously affected; replication of intracellular 2308 was detected at $48 \mathrm{~h}$ p.i. only. Both 2308 and 2308IK behaved similarly when the cells were infected with bacteria grown in iron-sufficient medium (inset). The complemented 2308IK(pBBRirr) strain was able to invade J774 cells; however, we were not confident of the accuracy of data obtained, since the infected cells were unstable. We are currently unable to explain the reason for this behaviour.

\section{Virulence in mice}

As the ability to survive inside eukaryotic cells is essential for the pathogenesis of $B$. abortus, we tested the virulence of the 
B. abortus strains in the mouse model. Two groups of 10 BALB/C mice were inoculated, one with 2308 and one with 2308IK, both grown in iron-deficient medium. As described in Methods, the number of viable bacteria recovered from the spleen of each mouse was determined at 7 and 21 days after inoculation, and the values obtained were $5 \cdot 2 \pm 0 \cdot 4$ and $4 \cdot 9 \pm 0 \cdot 3 \log$ (wild-type c.f.u. $\mathrm{ml}^{-1}$ ), and, $5 \cdot 2 \pm 0 \cdot 4$ and $5 \cdot 0 \pm 0 \cdot 5 \log \left(\right.$ mutant c.f.u. $\left.\mathrm{ml}^{-1}\right)$, respectively; thus, both strains showed similar behaviour. It is possible that the phenotype observed in vitro for 2308 was in some way compensated in vivo, at least in the experimental conditions used.

\section{DISCUSSION}

Bacteria compensate for iron limitation by inducing irontransport systems, and by reducing the cellular demand for iron. Irr seems to fulfil both functions in $B$. abortus. Previously, it has been shown that Irr down-regulates haem biosynthesis (Martínez et al., 2005). In this work, we demonstrate that $B$. abortus Irr upregulates siderophore production in $B$. abortus under conditions of iron limitation. The $B$. abortus irr secreted significantly less brucebactin than the wild-type strain. Further, detection of 2,3-DHBA from iron-limited bacterial cultures revealed that secretion of this siderophore was also reduced in the mutant. The deficiency in siderophore secretion in the mutant was restored by genetic complementation with a plasmid harbouring wild-type irr, indicating the involvement of Irr in the higher amounts of siderophores that accumulated in the extracellular medium of the wild-type cells. This could be the result of an induction in the biosynthesis of the catechols, and/or an induction of unknown secretion machinery. When the transcription of the B. abortus $d h b C$ was investigated, gene expression was decreased about twofold in the irr mutant compared with the wild-type under conditions of iron limitation. Therefore, Irr positively regulates 2,3-DHBA and brucebactin production; however, these results do not exclude regulation of secretion. Since the factor by which the secretion of the siderophores was affected was higher than that for transcription alone, it can be supposed that secretion was also induced. At this point, it must be considered that transcription and secretion are two different processes that do not necessarily respond with equal intensity to the same regulation.

In general, iron-uptake systems have been reported to be negatively regulated by Fur-like proteins that use ferrous ion as a cofactor. Thus, when iron is scarce, those regulated genes are de-repressed (Wandersman \& Delepelaire, 2004). The $d h b C E B A$ operon is expressed under conditions of iron limitation from two promoters containing Fur boxes (Bellaire et al., 2003); no evidence of its repression has yet been described. However, the fact that siderophores were secreted in the absence of the positive regulation in the $\mathrm{irr}$ mutant strongly suggests that the $\mathrm{dhbCEBA}$ operon is under dual regulation: it is repressed by an unknown regulator in the presence of iron, and induced by Irr in the absence of this metal. Using a gel retardation assay, we demonstrated that Irr is able to bind the upstream region of the operon. This region contains two putative Irr boxes (ICE motifs) (Rudolph et al., 2006), which are located just overlapping each of the two -10 promoters described in the $d h b C E B A$ operon. The DNA-Irr complexes were better resolved from naked DNA in agarose than they were in native polyacrylamide gels, possibly because they were large assembled complexes. In this regard, it must be kept in mind that Irr is able to dimerize in vitro, and the target DNA used in our gel mobility retardation assays presents two putative DNAbinding motifs. However, further analyses are needed to identify the DNA sequence that interacts with Irr, and whether it binds DNA as a dimer.

Irr is also implicated in oxidative stress resistance. While almost no $B$. abortus wild-type cells were recovered after exposure to hydrogen peroxide, more than $40 \%$ of $B$. abortus irr cells survived this oxidative stress, regardless of the physiological iron-deficient bacterial state. The $B$. abortus irr showed higher catalase activity than the wildtype. B. abortus catalase is transcriptionally regulated by OxyR, increasing its synthesis in response to hydrogen peroxide (Kim \& Mayfield, 2000; Kim et al., 2000). Beyond the induction of the gene, its product needs haem as a cofactor to be an active enzyme. Thus, the oxidative stress resistance of the irr mutant may be the result of extra haem molecules available to facilitate rapid formation of haemoproteins. It is also possible that Irr regulates the expression of catalase. In this regard, Irr could be a functional homologue of Bacillus subtilis Per (peroxide regulon regulator), which is a Fur-like protein that regulates both katA and a haem biosynthesis enzyme (Bsat et al., 1998). Similarly, a Campylobacter jejuni perR mutant has been described as being hyper-resistant to oxidative stress, with the $k a t A$ and the $a h p C$ genes being under derepression (van Vliet et al., 1999; Harvie et al., 2005). Per does not seem to be encoded in the genomes of B. abortus (Halling et al., 2005; Chain et al., 2005), Brucella melitensis (DelVecchio et al., 2002) and Brucella suis (Paulsen et al., 2002). A BLAST search (Altschul et al., 1990) using these translated genomes showed sequence homologies with very low values with a protein that belongs to the Irr family (Martínez et al., 2005), and with Fur. On the other hand, Irr is not present in Bac. subtilis or C. jejuni. In contrast, Bac. subtilis Per does not participate in the regulation of the Bacillus catecholic siderophores, while Bacillus Fur does (Chen et al., 1995).

The pathogenicity of Brucella spp. is based on their ability to survive and replicate inside macrophages. As one of the main bactericidal mechanisms used by macrophages is to produce hydrogen peroxide (Jiang \& Baldwin, 1993), it can be expected that a $B$. abortus strain with high catalase activity will offer more resistance to being killed by the macrophagelike J774 cells. The results presented here support this hypothesis: the $B$. abortus irr showed a better adaptation to the intracellular environment, recovering sooner than the wild-type from the initial loss of viability. The mechanisms 
against bacteria used by non-phagocytic cells are different; nonetheless, the irr mutant was able to successfully replicate during the first $24 \mathrm{~h}$ p.i. Wild-type cells lost viability during the same period; nevertheless, they replicated to the same extent as the irr mutant cells. It is noteworthy that the infection of HeLa and J774 cell lines with wild-type ironstarved $B$. abortus in the absence of fetal bovine serum was less efficient than when the cell lines are infected in nonlimiting conditions (Almirón et al., 2001); nonetheless, the invasion capability of the bacteria was not affected.

Previous reports have shown that the absence of catalase does not attenuate the virulence of B. melitensis in goats (Gee et al., 2004) or mice (Sangari \& Aguero, 1996). In a different way, 2,3-DHBA is not required for B. abortus virulence in mice (Bellaire et al., 1999), while it is considered a virulence factor in its natural host (Bellaire et al., 2003). Here, we present evidence that the combined effects of an increment in catalase activity and haem content, together with a decline in siderophore synthesis, do not alter the virulence of $B$. abortus in mice. This observation suggests that the observed in vitro phenotype of the irr mutant is not relevant in this animal model.

When the results are analysed together, it can be observed that differences between the $B$. abortus wild-type and $B$. abortus irr phenotypes are evident after short time periods, as if they are reflecting a delay in the wild-type response to the imposed stress condition. This lag may correspond with the time needed for $B$. abortus to increase its haem level through iron uptake and haem biosynthesis, or through haem-uptake systems. Once the goal of increasing the haem level is achieved, the stability of Irr is affected (Martínez et al., 2005; Qi \& O'Brian, 2002; Qi et al., 1999). Thus, the wildtype phenotype in the iron-limited condition will resemble that of the irr mutant, resulting in an improved stress response.

To conclude, Irr directly induces iron assimilation to ensure haem biosynthesis in B. abortus. Haem biosynthesis is essential for the intracellular survival of this animal pathogen (Almirón et al., 2001). In a similar way, Bradyrhizobium japonicum Irr coordinates high-affinity iron-uptake systems with the synthesis of haem, a molecule indispensable to efficiently nodulate soybeans (Hamza et al., 1998). Whilst Brucella and Bradyrhizobium belong to the $\alpha-2$ Proteobacteria, and Irr is confined to this group, it is possible that the regulation exerted by Irr represents a common strategy for intracellular adaptation among plant symbionts and animal pathogens (LeVier et al., 2000; Ugalde, 1999).

\section{ACKNOWLEDGEMENTS}

We thank A. Merás for technical assistance with the use of HPLC, and $\mathrm{P}$. Briones for computational assistance. This work was supported by grants PICT 01-6580 to M. A., and PICT 01-6565 to R. A. U., both from Agencia Nacional de Promoción Científica y Tecnológica, Argentina.

\section{REFERENCES}

Almirón, M., Link, A. J., Furlong, D. \& Kolter, R. (1992). A novel DNA-binding protein with regulatory and protective roles in starved Escherichia coli. Genes Dev 6, 2646-2654.

Almirón, M., Martínez, M., Sanjuan, N. \& Ugalde, R. A. (2001). Ferrochelatase is present in Brucella abortus and is critical for its intracellular survival and virulence. Infect Immun 69, 6225-6230.

Altschul, S. F., Gish, W., Miller, W., Myers, E. W. \& Lipman, D. J. (1990). Basic local alignment search tool. J Mol Biol 215, 403-410.

Beers, R. F., Jr \& Sizer, I. W. (1952). A spectrophotometric method for measuring the breakdown of hydrogen peroxide by catalase. J Biol Chem 195, 133-140.

Bellaire, B. H., Elzer, P. H., Baldwin, C. L. \& Roop, R. M., II (1999). The siderophore 2,3-dihydroxybenzoic acid is not required for virulence of Brucella abortus in Balb/c mice. Infect Immun. 67, 2615-2618.

Bellaire, B. H., Elzer, P. H., Hagius, S., Walker, J., Baldwin, C. L. \& Roop, R. M., II (2003). Genetic organization and iron-responsive regulation of the Brucella abortus 2,3-dihydroxybenzoic acid biosynthesis operon, a cluster of genes required for wild-type virulence in pregnant cattle. Infect Immun 71, 1794-1803.

Bradford, M. M. (1976). A rapid and sensitive method for the quantitation of microgram quantities of protein utilizing the principle of protein-dye binding. Anal Biochem 72, 248-254.

Bsat, N., Herbig, A., Casillas-Martínez, L., Setlow, P. \& Helmann, J. D. (1998). Bacillus subtilis contains multiple Fur homologues: identification of the iron uptake (Fur) and peroxide regulon (PerR) repressors. Mol Microbiol 29, 189-198.

Bullen, J. J. \& Griffiths, E. (1999). Iron and Infection: Molecular, Physiological and Clinical Aspects, pp. 1-26. Chichester, UK: Wiley.

Chain, P. S., Comerci, D. J., Tolmasky, M. E. \& 7 other authors (2005). Whole-genome analyses of the speciation events in the pathogenic Brucellae. Infect Immun 73, 8353-8361.

Chen, L., Keramati, L. \& Helmann, J. D. (1995). Coordinate regulation of Bacillus subtilis peroxide stress genes by hydrogen peroxide and metal ions. Proc Natl Acad Sci U S A 92, 8190-8194.

DelVecchio, V. G., Kapatral, V., Redkar, R. J. \& 22 other authors (2002). The genome sequence of the facultative intracellular pathogen Brucella melitensis. Proc Natl Acad Sci U S A 99, 443-448.

Frustaci, J. M., Sangwan, I. \& O'Brian, M. R. (1991). Aerobic growth and respiration of a delta-aminolevulinic acid synthase (hemA) mutant of Bradyrhizobium japonicum. J Bacteriol 173, 1145-1150.

Gee, J. M., Kovach, M. E., Grippe, V. K., Hagius, S., Walker, J. V., Elzer, P. H. \& Roop, R. M., II (2004). Role of catalase in the virulence of Brucella melitensis in pregnant goats. Vet Microbiol 102, 111-115.

Gonzalez Carrero, M. I., Sangari, F. J., Aguero, J. \& Garcia Lobo, J. M. (2002). Brucella abortus strain 2308 produces brucebactin, a highly efficient catecholic siderophore. Microbiology 148, 353-360.

Halling, S. M., Peterson-Burch, B. D., Bricker, B. J., Zuerner, R. L., Qing, Z., Li, L. L., Kapur, V., Alt, D. P. \& Olsen, S. C. (2005). Completion of the genome sequence of Brucella abortus and comparison to the highly similar genomes of Brucella melitensis and Brucella suis. J Bacteriol 187, 2715-2726.

Hamza, I., Chauhan, S., Hassett, R. \& O'Brian, M. R. (1998). The bacterial Irr protein is required for coordination of heme biosynthesis with iron availability. J Biol Chem 273, 21669-21674.

Harvie, D. R., Vilchez, S., Steggles, J. R. \& Ellar, D. J. (2005). Bacillus cereus Fur regulates iron metabolism and is required for full virulence. Microbiology 151, 569-577. 
Jiang, X. \& Baldwin, C. L. (1993). Iron augments macrophagemediated killing of Brucella abortus alone and in conjunction with interferon-gamma. Cell Immunol 148, 397-407.

Kim, J. A. \& Mayfield, J. (2000). Identification of Brucella abortus OxyR and its role in control of catalase expression. J Bacteriol 182, 5631-5633.

Kim, J. A., Sha, Z. \& Mayfield, J. E. (2000). Regulation of Brucella abortus catalase. Infect Immun 68, 3861-3866.

LeVier, K., Phillips, R. W., Grippe, V. K., Roop, R. M., II \& Walker, G. C. (2000). Similar requirements of a plant symbiont and a mammalian pathogen for prolonged intracellular survival. Science 287, 2492-2493.

Litwin, C. M. \& Calderwood, S. B. (1993). Role of iron in regulation of virulence genes. Clin Microbiol Rev 6, 137-149.

Lopez-Goñi, I., Moriyón, I. \& Neilands, J. B. (1992). Identification of 2,3-dihydroxybenzoic acid as a Brucella abortus siderophore. Infect Immun 60, 4496-4503.

Martínez, M., Ugalde, R. A. \& Almirón, M. (2005). Dimeric Brucella abortus Irr protein controls its own expression and binds haem. Microbiology 151, 3427-3433.

Miller, J. H. (1992). A Short Course in Bacterial Genetics. Cold Spring Harbor, NY: Cold Spring Harbor Laboratory.

Paulsen, I. T., Seshadri, R., Nelson, K. E., Eisen, J. A., Heidelberg, J. F., Read, T. D., Dodson, R. J. \& 28 other authors (2002). The Brucella suis genome reveals fundamental similarities between animal and plant pathogens and symbionts. Proc Natl Acad Sci U S A 99, $13148-13153$.

Qi, Z. \& O'Brian, M. R. (2002). Interaction between the bacterial iron response regulator and ferrochelatase mediates genetic control of heme biosynthesis. Mol Cell 9, 155-162.

Qi, Z., Hamza, I. \& O’Brian, M. R. (1999). Heme is an effector molecule for iron-dependent degradation of the bacterial iron response regulator (Irr) protein. Proc Natl Acad Sci U S A 96, 1305613061.

Roop, M. R., II, Bellaire, B. H., Anderson, A. J. \& Paulley, J. T. (2004). Iron metabolism in Brucella. In Brucella: Molecular and Cellular Biology, pp. 243-262. Edited by I. Lopez-Goni \& I. Moriyón. Pamplona: Horizon Bioscience.

Rudolph, G., Semini, G., Hauser, F., Lindemann, A., Friberg, M., Hennecke, H. \& Fischer, H. M. (2006). The Iron control element, acting in positive and negative control of iron-regulated Bradyrhizobium japonicum genes, is a target for the Irr protein. J Bacteriol 188, 733-744.

Sangari, F. J. \& Aguero, J. (1996). Molecular basis of Brucella pathogenicity: an update. Microbiologia 12, 207-218.

Schwyn, B. \& Neilands, J. B. (1987). Universal chemical assay for the detection and determination of siderophores. Anal Biochem 160, $47-56$.

Sha, Z., Stabel, T. J. \& Mayfield, J. E. (1994). Brucella abortus catalase is a periplasmic protein lacking a standard signal sequence. J Bacteriol 176, 7375-7377.

Smith, L. D. \& Ficht, T. A. (1990). Pathogenesis of Brucella. Crit Rev Microbiol 17, 209-230.

Ugalde, R. A. (1999). Intracellular lifestyle of Brucella spp. Common genes with other animal pathogens, plant pathogens, and endosymbionts. Microbes Infect 1, 1211-1219.

van Vliet, A. H., Baillon, M. L., Penn, C. W. \& Ketley, J. M. (1999). Campylobacter jejuni contains two fur homologs: characterization of iron-responsive regulation of peroxide stress defense genes by the PerR repressor. J Bacteriol 181, 6371-6376.

Wandersman, C. \& Delepelaire, P. (2004). Bacterial iron sources: from siderophores to hemophores. Annu Rev Microbiol 58, 611-647. 